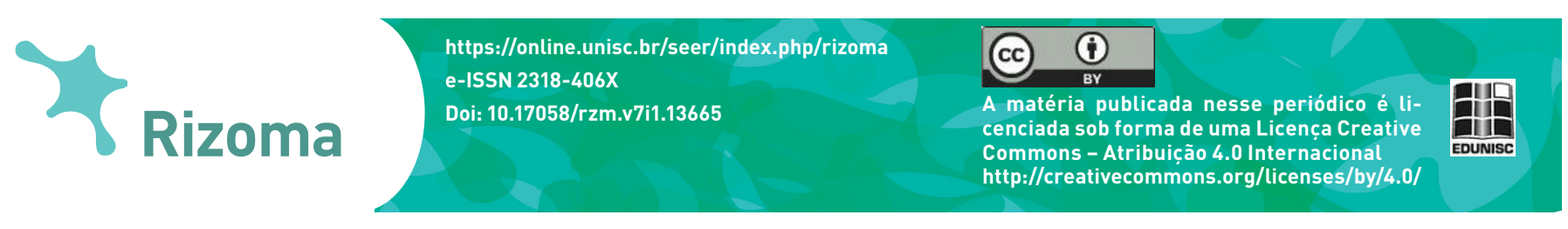

\title{
Resenha
}

\section{La conectividad en tiempos de pos-verdad}

\section{José van Dijck}

Originalmente publicado en 2013, y luego traducido y publicado por la editorial siglo XXI (2016) en la colección Sociología y Política de la serie Rumbos Teóricos.

Existen diversas investigaciones que se han desarrollado en las últimas décadas sobre las nuevas tecnologías de la información, y específicamente sobre las plataformas de interacción social que se han desarrollado dentro de la denominada web 2.0. No obstante, la investigación de van Dijck tiene la virtud de presentar una reflexión integral, desde un enfoque sociológico, sobre como estas tecnologías se han integrado en nuestra vida social y se han sedimentado como infraestructuras que han reorganizado nuestra socialidad. Es en este sentido, es pertinente discutir a van Dijck para comprender la manera en la que ciertos mensajes que circulan en estas plataformas, específicamente las noticias falsas o Fake News, síntomas de una era de pos-verdad pueden infiltrarse de manera generalizada en nuestro cotidiano. Entender esta nueva forma de estructurar la sociabilidad puede ayudarnos a entender estos fenómenos sociales y comunicacionales con diversas consecuencias para nuestras sociedades. Por ello, seguiré la pista de reflexión sobre la circulación de noticias falsas en nuestro cotidiano para discutir los diferentes aportes que la profesora holandesa de medios y sociedad digital en la Universidad de Utrecht realiza para este debate de actualidad.

De manera general, La cultura de la conectividad plasma la trayectoria de investigación de la autora en el campo denominado de los "nuevos medios de comunicación”. El libro se estructura en ocho capítulos. El primero traza la historia de lo que la autora denomina "medios conectivos" (entendidos como aplicativos digitales de interconexión) y la manera en la que
Sofia Isabel Vizcarra Vizcarra Castillo

${ }^{1}$ Pós-doutoranda no Departamento de Economia e Relaçoes Internacionais no Projeto sobre Cultura Política e a Cultura Mundial de Valores da Universidade Federal de Rio Grande do Sul (UFRGS). Doutora em Sociologia e mestre em Ciência Política pela Pontifícia Universidade Católica do Peru (PUCP) e Mestre em Segurança Internacional pelo Institut d'études Politiques de Paris - Sciences Po. Professora do departamento de Ciencias Socias da PUCP. 
estos producen socialidad. El segundo capítulo propone un marco analítico innovador que une dos perspectivas teóricas diferentes: la economía política y la teoría del actor-red con el fin de analizar tanto la dimensión socio-económica como socio-técnica de estos medios. Los cinco capítulos siguientes abordan el análisis de cinco medios conectivos específicos - Facebook, Twitter, Flickr, Youtube y Wikipedia - según seis dimensiones: el modelo de negocio, la estructura de gobierno, los regimenes de propiedad, los usuarios y sus hábitos de uso, la tecnología empleada y los contenidos que circulan. Este análisis le permite plantear, en el último capítulo, un modelo de ecosistema de los diferentes conectivos bajo la tensión de dos demandas contradictorias: las demandas de los usuarios por mayor libertad y funcionalidad y aquella de las corporaciones detrás de las plataformas que buscan hacer rentables sus inversiones.

Si bien, las reflexiones de van Dijck sobre estas tensiones son pertinentes, creo que en el caso del análisis de fenómenos relacionados a la mediatización así como a la difusión de contenidos relacionados a la pos-verdad, específicamente la difusión de Fake News, es pertinente explorar detalladamente la propuesta conceptual de la autora sobre la cultura de la conectividad. Este concepto permite abarcar el conjunto de procesos sociales, incluyendo los comunicacionales, que ocurren en la web 2.0 así como fuera de ella. Esto significa que es un concepto integral que por una parte, analiza la vida social en los diferentes medios conectivos a partir de los procesos específicos a cada uno de ellos. Por otra parte, analiza los procesos sociales virtuales y fuera de la web como un flujo continuo de interacciones, sin distinción entre "mundo real" y "mundo virtual", ya que, como lo afirma la autora nuestra socialidad incluye "tanto la conexión social (humana) como la conectividad (automática)" (DIJCK, 2016, p. 30) siendo indisociables para su comprensión.

Van Dijck entiende que los cambios que introducen los medios conectivos no son simplemente cambios en las formas de comunicación, sino que generan una verdadera "batalla cultural por imponer un nuevo orden regulatorio para la comunicación y socialización online" (2016, p. 108). Este nuevo orden regulatorio implica la adopción y promoción de nuevos valores sociales así como prácticas tecnológicas que promueven tanto la conexión como la conectividad. La reflexión sobre los valores promovidos es clave para comprender de que manera los contenidos que circulan en los diferentes medios conectivos son internalizados y generan efectos más allá del mundo virtual. A lo largo del libro, van Dijck nos propone pensar en los valores promovidos por cada 


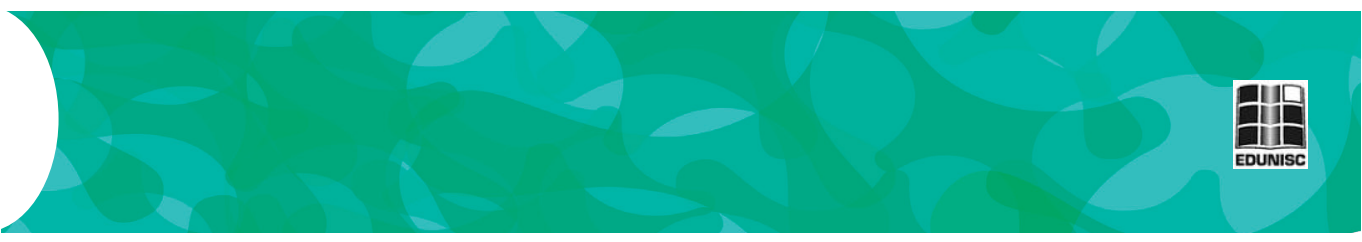

uno de los aplicativos que analiza así como las características tecnológicas que les dan soporte, el resumen de este análisis se presenta en la tabla $n^{\circ} 1$.

Tabla 1 - Valores promovidos y soporte tecnológico de diferentes medios conectivos

\begin{tabular}{|l|l|l|}
\hline Medio conectivo & Valores sociales promovidos & \multicolumn{1}{|c|}{ Configuración del soporte tecnológico } \\
\hline Facebook & $\begin{array}{l}\text { "compartir", "gustar", "hacer } \\
\text { amigos" }\end{array}$ & $\begin{array}{l}\text { Configuración por default (algoritmos específicos } \\
\text { que favorecen ciertas interacciones y botones } \\
\text { correspondientes a cada uno de estos valores) }\end{array}$ \\
\hline Twitter & "seguir"y "marcar tendencia" & $\begin{array}{l}\text { Funciones de trending topics (TT), retweets (RT) } \\
\text { y hashtags (\#) }\end{array}$ \\
\hline Flickr & "comunidad"y "memoria" & $\begin{array}{l}\text { Configuración por default (a partir de las } \\
\text { funciones compartir y favoritos) }\end{array}$ \\
\hline Youtube & $\begin{array}{l}\text { "homecasting"y "compartir } \\
\text { videos" }\end{array}$ & $\begin{array}{l}\text { Funciones favoritos y desarrollo de la tecnología } \\
\text { de streaming (a partir de lo que fue la tecnología } \\
\text { Peer-to-peer P2P) }\end{array}$ \\
\hline Wikipedia & "conocimiento"y "neutralidad" & $\begin{array}{l}\text { Arquitectura anárquica basada en contenidos } \\
\text { colaborativos. }\end{array}$ \\
\hline
\end{tabular}

Fuente: Elaboración propia a partir de van Dijck, 2016.

Así, a pesar de sus diferencias y diferentes niveles de éxito en sedimentar la aceptación de estos valores, se puede observar que la mayoría de plataformas se centrar en el usuario. Los valores parten por lo tanto del tipo de interacciones que el usuario puede desarrollar a partir de ellas. Todas promueven la conectividad como valor, haciendo así deseable y positivo interactuar a través de los diferentes aplicativos. Sin embargo, las tecnologías que dan soporte a estos medios conectivos no son neutras, como lo señala la autora. Por el contrario, los componentes socio-técnicos de estos medios no responden únicamente a los usuarios sino a las presiones de las corporaciones propietarias de la mayoría de estos medios conectivos que tratan de maximizar el lucro de la conectividad que ofrecen. En ese sentido, los intereses que promueven los cambios tecnológicos y estructuran nuestra socialidad deben ser visibilizados en sus características e intencionalidad. Para ellos, van Dijck propone prestar particular atención a conceptos como (meta)datos, algoritmo, protocolo, interface y default que permiten desmontar la armazón tecnológica por tras de estos medios conectivos.

Así por ejemplo, el estudio de la circulación de Fake News en medios conectivos como Facebook debe integrar el análisis 
de los algoritmos que privilegian las noticias que los individuos reciben en sus muros. ¿Por qué? Porque estos algoritmos se basan en la afinidad y familiaridad, por lo tanto tienden a mostrar publicaciones de personas con las que se concuerda o al menos en quienes se confía, y por lo tanto facilitar la aceptación de las Fake News. Ello podría provocar una mayor exposición a las noticias falsas de personas cuyos círculos sociales próximos comparten frecuentemente este tipo de noticias. Además, el análisis sobre los valores de la cultura de la conectividad también permiten explicar, en parte, porque la escala de difusión de este tipo de noticias ha aumentado de manera exponencial en los últimos años. Como vimos en la tabla $\mathrm{n}^{\mathrm{o}} 1$, varios medios conectivos promueven "compartir" como valor, si bien cada uno lo entiende de manera diferentes, el efecto es aumentar el número de interacciones y conexiones, lo que promueve la circulación de todo tipo de contenidos, incluyendo las noticias falsas. No obstante, como la conexión es la base del lucro de varias de estas plataformas, las medidas para prevenir la diseminación de estos contenidos relacionadas a los soportes tecnológicos de estos medios tardaron en ser discutidas. A pesar de que los algoritmos y configuraciones por default tuvieron un rol fundamental para la propagación de noticias falsas (así como de otro tipo de contenidos con impactos negativos en la vida social, como los discursos de odio), la discusión sobre cambiar su configuración para evitar ciertos comportamientos (y por ende ciertas conexiones) no se han dado a la misma velocidad que la propagación de estos fenómenos. Así, la reflexión propuesta por van Dijck sobre conexión y conectividad como dos caras de una misma moneda nos lleva a preguntar sobre la responsabilidad de los actores detrás de la conectividad en la sedimentación de la era de la pos-verdad.

No obstante, el balance de van Dijck sobre los medios conectivos no es totalmente negativa. Una vez que entendemos que los medios conectivos constituyen un eco-sistema imbricado en un contexto social, económico, político y cultural más amplio, entonces entendemos que es un fenómeno sobre el cual nuestra acción tiene un impacto directo. Si bien las relaciones de poder que estructuran este eco-sistema distan de ser simétricas, ellos no significan que sus valores y estructuras sean fijas. A pesar de que en La cultura de la conectividad, las evidencias sobre los impactos en la vida social cotidiana no necesariamente en el primer plano, al centrarse principalmente en el planteamiento de un modelo teórico de análisis de los medios conectivos, ellos no significa que este tema no esté en el centro de la reflexión de la autora. Así, van Dijck continua reflexionando sobre las interacciones entre el mundo 

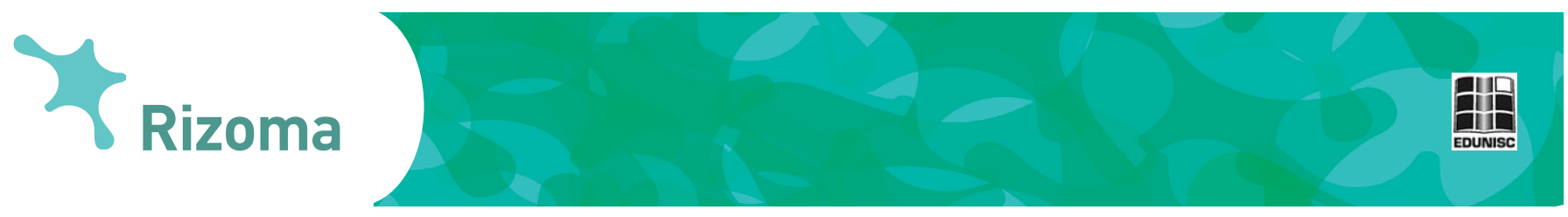

tecnológico y los procesos sociales, centrándose específicamente en los valores sociales que circulan ya no solo en los medios conectivos sino en las diferentes plataformas de servicios que parecen ganar terreno en varios países. Ese es el objeto de su nuevo libro junto con Thomas Poell y Martjinde Waal (2018) en el que proponen ampliar su análisis sobre las normas y valores de la conectividad para centrarse en los valores públicos y las tensiones entre interés público y privado en lo que ellos denominan "la sociedad de las plataformas". Esperemos que pronto sea traducido al español y portugués. Vale la pena, continuar siguiendo el trabajo de la autora para seguir informando teóricamente el debate sobre medios conectivos y pos-verdad. 